\title{
Dynamic 3D MR-Defecography
}

\section{Dynamische 3D-MR-Defäkografie}

\section{Abstract \\ $\nabla$}

Epidemiological studies have estimated the incidence of chronic constipation to be up to $27 \%$ of the general population. The gold standard to evaluate affected patients is the dynamic entero-colpo-cysto-defecography. In the clinical routine 2 D MR-defecography is also performed, but only one to three $2 \mathrm{D}$ slices at a temporal footprint of about one second are acquired. To improve the detection of lateral localized pathologies, we developed and implemented dynamic 3D MR-defecography. Each 3 D block consisted of seven slices with an inplane spatial resolution of $1.3 \times 1.3 \mathrm{~mm}^{2}$ to $2.3 \times 2.3 \mathrm{~mm}^{2}$ and an image update rate between $0.8 \mathrm{~s}$ and $1.3 \mathrm{~s}$. We used a fast bSSFP sequence with a modified stack-of-stars sampling scheme for data acquisition and a modified FISTA compressed sensing algorithm to reconstruct the undersampled datasets. We performed a study including 6 patients to optimize the acquisition parameters with respect to image quality.

\section{Zusammenfassung}

Epidemiologische Studien schätzen die Inzidenz chronischer Obstipation auf bis zu $27 \%$ der Gesamtbevölkerung. In der Regel wird zur Untersuchung betroffener Patienten die Entero-Colpo-Cysto-Defäkografie verwendet. Auch die 2D-MR-Defäkografie wird im klinischen Alltag angewendet, jedoch wird hier lediglich die Dynamik in ein bis drei 2D-Schichten dargestellt. Die Evaluation von lateral gelegenen Pathologien kann hierdurch beeinträchtigt sein. Deshalb haben wir eine 3D-MR-Defäkografie entwickelt und implementiert. Jeder 3D-Block bestand aus sieben Schichten mit einer räumlichen Auflösung zwischen $1,3 \times 1,3 \mathrm{~mm}^{2}$ und $2,3 \times 2,3 \mathrm{~mm}^{2}$. Die Bildaktualisierungsrate lag zwischen $0,8 \mathrm{~s}$ und $1,3 \mathrm{~s}$. Wir verwendeten für die Datenakquisition eine modifizierte Stack-of-Stars bSSFP-Sequenz und für die Datenrekonstruktion einen modifizierten FISTAcompressed-sensing-Algorithmus. Für die Optimierung der Akquisitionsparameter hinsichtlich der Bildqualität haben wir eine Studie mit 6 Patienten durchgeführt.

\section{Introduction \\ $\nabla$}

Epidemiological studies have shown that the incidence of chronic obstructive diseases is up to $27 \%$ of the general population. The gold standard to evaluate affected patients is the radiographic method of entero-colpo-cysto defecography (ECCD) (Cappabianca et al. Int J Colorectal Dis 2011; 26: 1191 -1196). Two major disadvantages of ECCD are the application of ionizing radiation in the pelvic floor region and the unpleasant measurement procedure for patients. Contrast agent has to be administered to the rectum, bladder, vagina and the small intestine of the patient. In the clinical routine, $2 \mathrm{D}$ MR-defecography is often applied as a supplementary method. MR-defecography only requires rectal filling with sonographic gel as the contrast agent and no ionizing radiation has to be administered in the pelvic floor region. However, imaging of the fast dynamic and non-periodic defecation process is challenging due to the lengthy data acquisition in MR imaging. Thus, with standard methods only a very limited number of $2 \mathrm{D}$ slices can be sampled to still achieve a sufficient temporal resolution for the single slices.

Consequently, both ECCD and MR-defecography feature restricted spatial coverage and therefore lack information about the lateral extent of obstruction-related pathologies like rectoceles or intussusceptions. Nevertheless, newer sampling trajectories and acceleration techniques in MR imaging have the potential to overcome the limitations named above. While parallel imaging is already widely used in the clinical routine, the compressed sensing (CS) technique (Lustig et al. Magn Reson Med 2007; 58: $1182-1195$ ) is still restricted to research. Nevertheless, CS has been proven to allow reconstruction of highly undersampled data, especially in acquisitions of dynamic processes, thereby significantly accelerating the scan time. CS uses information about the object to be imaged, which is already known before the measurement, and includes this prior knowledge as a constraint in the image reconstruction process. By doing this, less data is needed to obtain images free of under- sampling artifacts. Therefore, it is possible to acquire more slices within a certain amount of time and thereby cover the dynamics of the defecation process not only in single $2 \mathrm{D}$ slices but in a complete $3 \mathrm{D}$ volume.

In this paper, we propose a $3 \mathrm{D}$ MR-defecography setup that uses an extended radial bSSFP stack-of-stars (Wech T. Fortschr Röntgenstr 2014; 186: 37 - 41) imaging sequence for data acquisition and a modified "Fast Iterative Shrinkage Threshold Algorithm - FISTA" (Beck A. et al. SIIMS 2009; 2: 183-202) compressed sensing algorithm for data reconstruction. We applied this setup to 6 female patients in order to optimize the imaging protocol with respect to the sampling strategy as well as the spatial and temporal resolution.

\section{Materials and Methods}

$\nabla$

All measurements were performed on $3 \mathrm{~T}$ whole-body systems (MAGNETOM Prisma and MAGNETOM Skyra, Siemens Healthcare $\mathrm{GmbH}$, Erlangen) equipped with a 32channel body array coil. All reconstruction algorithms were implemented using Matlab 2014b (The Mathworks, Natick, MA, USA).

Data acquisition was performed using a sagittal 3D bSSFP stack-of-stars imaging sequence. This technique uses standard phase encoding in the $\mathrm{k}_{\mathrm{z}}$-direction and $\mathrm{a}$ radial sampling scheme in each of the $\mathrm{k}_{\mathrm{x}}{ }^{-}$ $\mathrm{k}_{\mathrm{y}}$-planes. The most straightforward implementation of the stack-of-stars trajectory acquires each $\mathrm{k}_{\mathrm{z}}$-partition of a $3 \mathrm{D}$ block one after another and with the same number of spokes in each partition ( $\bullet$ Fig. 1a). In addition to this version, two supplementary features were implemented. The first one is density weighting (DW), which is shown in 1 Fig. 1b. The number of spokes varies between the different partitions of the $3 \mathrm{D}$ block and increases towards the central partition. We acquired seven partitions from $\mathrm{k}_{z, \min }=-3$ to $\mathrm{k}_{\mathrm{z} \text {,max }}=3$ and applied two different undersampling patterns for $\mathrm{k}_{\mathrm{x}, \mathrm{y}}$. Compared to full Nyquist sampling at $\mathrm{k}_{\mathrm{x}, \mathrm{y}}=\max$, the undersampling factors $\mathrm{R}$ from the center partition $\mathrm{k}_{\mathrm{z}}=0$ to the outer partitions were $\mathrm{R}=3,4,5,6$ (DW sampling 1) and $\mathrm{R}=3,4,8,10$ (DW sampling 2), respectively. The second feature is view sharing, in which the order of the acquired partitions is changed from linear to a rearranged order while the central partition is sampled more often. The corresponding sampling scheme is shown in - Fig. 1c. In every partition the spokes are 


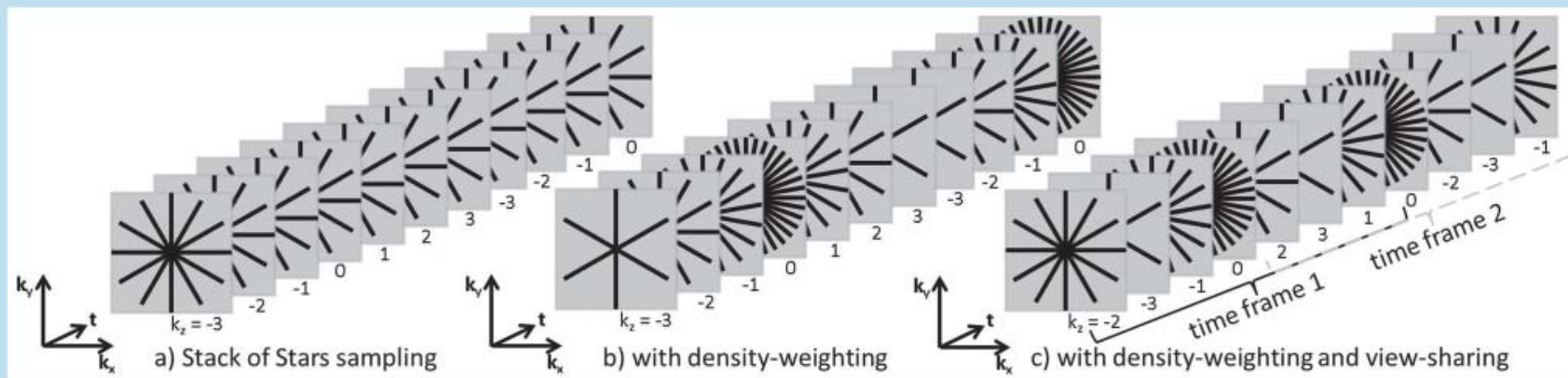

Fig. 1 a Standard stack-of-stars sampling scheme. Each time frame (which is equivalent to one $3 \mathrm{D}$ volume) consists of seven partitions ( $k z=-3 . . .3)$ that are measured one after another. $\mathbf{b}$ In case of density weighting, the number of acquired spokes increases towards the center partition. c Additional view sharing that acquires the partitions in a rearranged order and acquires the center partition $\mathrm{kz}=0$ more often was implemented. Therefore, the number of reconstructed time frames is almost doubled.
Abb. 1 a Standard „Stack-of-Stars“ Abtastungsschema. Jedes 3D-Volumen besteht aus sieben Partitionen ( $k z=-3 \ldots 3$ ), welche der Reihe nach gemessen werden. b Beim „density-weighting“ nimmt die Anzahl an gemessenen radialen Linien zur Mitte hin zu. c Im Gegensatz zum Standardschema werden beim „view-sharing“ die einzelnen Partitionen nicht linear, sondern in einer umsortierten Reihenfolge gemessen. Dabei wird die mittlere Partition $\mathrm{kz}=0$ zweimal pro 3D-Volumen gemessen, wodurch nahezu doppelt so viele 3D-Volumen („time frames“) rekonstruiert werden können.

Table 1 Measurement parameters of all 6 female patients and their age.

Tab. 1 Messparameter aller 6 weiblichen Patienten und deren Alter.

\begin{tabular}{|llllllllll} 
patient & age & $\begin{array}{l}\text { temporal } \\
\text { res./s }\end{array}$ & $\begin{array}{l}\text { update } \\
\text { rate/s }\end{array}$ & matrix size & FoV/mm & voxel size/mm $\mathbf{m}^{3}$ & TR/ms & TE/ms & DW sampling \\
\hline 1 & 68 & 1.6 & 1.6 & $256 \times 256 \times 8$ & $280 \times 280 \times 32$ & $2.2 \times 2.2 \times 4$ & 3.1 & 1.5 & 0 \\
\hline 2 & 62 & 1.6 & 1.6 & $256 \times 256 \times 8$ & $300 \times 300 \times 32$ & $2.3 \times 2.3 \times 4$ & 2.9 & 1.4 & 0 \\
\hline 3 & 70 & 2.1 & 1.3 & $384 \times 384 \times 7$ & $270 \times 270 \times 56$ & $1.4 \times 1.4 \times 8$ & 3.4 & 1.7 & 1 \\
\hline 4 & 80 & 1.2 & 0.8 & $256 \times 256 \times 7$ & $256 \times 256 \times 42$ & $2.0 \times 2.0 \times 6$ & 3.0 & 1.5 & 1 \\
\hline 5 & 26 & 2.0 & 1.3 & $448 \times 448 \times 7$ & $300 \times 300 \times 35$ & $1.3 \times 1.3 \times 5$ & 3.5 & 1.8 & 2 \\
\hline 6 & 54 & 2.0 & 1.3 & $448 \times 448 \times 7$ & $300 \times 300 \times 35$ & $1.3 \times 1.3 \times 5$ & 3.5 & 1.8 & 2 \\
\hline
\end{tabular}

Temporal and spatial resolution parameters, the repetition time (TR) and echo time (TE), as well as the applied DW sampling scheme are shown.

Parameter zur zeitlichen und räumlichen Auflösung, Repetitionszeit (TR) und Echozeit (TE), sowie das verwendete DW-Sampling-Schema sind dargestellt.

sampled in linear order and every second spoke is measured in the reversed direction to compensate for eddy currents.

Data were initially gridded onto a Cartesian grid using the parallel imaging technique of self-calibrated GRAPPA operator gridding (GROG). Subsequently, data reconstruction was performed using a compressed sensing technique, which enforces sparsity in the spatial wavelet domain. In general, this optimization problem can be mathematically expressed by

$\min _{m}\left\|F_{\mathrm{u}} m-y\right\|_{2}^{2}+\lambda\|\Psi m\|_{1}$

where $m$ represents the image data to be reconstructed and $\mathrm{y}$ is the undersampled k-space measurement. The operator $\mathrm{Fu}_{\mathrm{as}}$ applies a Fourier transform and masks k-space data not sampled. The first term of the equation thus enforces data consistency between the current solution and the undersampled acquisition. The second term enforces the reconstructed image to be sparse in the wavelet domain. $\psi$ represents a wavelet transform operator which is applied to the solution $\mathrm{m}$.
The regularization parameter $\lambda$ realizes a trade-off between data consistency and sparsity in the wavelet transform domain. To effectively perform the optimization, we implemented a modified FISTA algorithm in analogy to Wech $\mathrm{T}$. et al. (Wech T. et al. IEEE Transactions on Medical Imaging 2015; 35: 912 -920).

The described acquisition and reconstruction scheme was applied to 6 female patients. The study was approved by the ethics committee of our institution and written informed consent was obtained from all patients participating in the study. All patients underwent a clinically indicated 2D MR-defecography examination. The MR-defecography protocol consisted of three static high-resolution 2D TSE sequences for scouting (FOV: $350 \times 350 \mathrm{~mm}^{2}$, voxel size: $0.7 \times 0.7 \mathrm{~mm}^{2}$, slice thickness: $3 \mathrm{~mm}$, flip angle: $150^{\circ}$, TR $=3780 \mathrm{~ms}$, $\mathrm{TE}=82 \mathrm{~ms}$ ) and a dynamic $2 \mathrm{D}$ examination was performed thereby acquiring three separated sagittal $2 \mathrm{D}$ slices using a bSSFP sequence (FOV: $320 \times 320 \mathrm{~mm}^{2}$, voxel size: $0.6 \times 0.6 \mathrm{~mm}^{2}$, slice thickness $=8 \mathrm{~mm}$, flip angle: $50^{\circ}, \mathrm{TR}=3.48 \mathrm{~ms}, \mathrm{TE}=1.55 \mathrm{~ms}$ ). The rectum was contrasted with $200 \mathrm{ml}$ of sonographic gel.

After this $2 \mathrm{D}$ examination the patient's rectum was refilled with sonographic gel and the proposed 3 $\mathrm{D}$ examination scheme was applied. During both dynamic measurements the patients were asked to strain and squeeze and then to evacuate the rectum. The imaging parameters of the $3 \mathrm{D}$ sequence of all patients are shown in 0 Table 1. We used an in-plane spatial resolution from $1.3 \times 1.3 \mathrm{~mm}^{2}$ to $2.3 \times 2.3 \mathrm{~mm}^{2}$ with a slice thickness of 4 to $8 \mathrm{~mm}$. Depending on the spatial resolution and on the density weighting scheme, we obtained a temporal resolution from 1.2 to 2.1 seconds. By applying view sharing, the respective time frames were updated every $0.8 \mathrm{~s}$ to $1.3 \mathrm{~s}$. For the first two patients a standard stack-ofstars sampling scheme ( $\bullet$ Fig. 1a) was used. The undersampling factor in all partitions was $\mathrm{R}=4$ and eight partitions were measured in these cases, while for all other patients the additional features of 
density weighting and view sharing were applied for seven partitions. Patients 3 and 4 were measured using DW sampling 1 with lower undersampling factors in the outer partitions and patients 5 and 6 were examined using DW sampling 2 with higher undersampling factors in the outer partitions. The flip angle was between $39^{\circ}$ and $42^{\circ}$.

\section{Results}

\section{$\nabla$}

The compressed sensing reconstructed datasets allowed examination of the defecation process within the whole acquired $3 \mathrm{D}$ volume. 0 Fig. 2 shows one central slice of a single time point for each patient to allow comparison of the different sampling schemes. The sagittal view visualizes the rectum of the six patients that was filled with $200 \mathrm{ml}$ of sonographic gel.

All patients had a small $(<2 \mathrm{~cm})$, medium $(2-4 \mathrm{~cm})$ or large $(>4 \mathrm{~cm})$ anterior rectocele. The images acquired without density weighting and without view sharing (DW sampling 0 , patients $1 \& 2$ ) indeed show the pathology. However, compared to the images of patients 5 and 6 , significant blurring due to the low spatial resolution of the images impairs diagnosis. The second sampling scheme (DW sampling 1, patients 3 \& 4) resulted in images that also allow detection of the rectocele in each case, but an increased level of incoherent artifacts (see white arrow) remains after application of the proposed reconstruction method. The results obtained using DW sampling 2 feature the best image quality with high spatial resolution and low artifact power.

The top of $\bullet$ Fig. 3 depicts a dynamic image series exemplarily for slice five in patient 5 . The series clearly shows the evolution of a small anterior rectocele. The series presented at the bottom of $\bullet$ Fig. 3 , 먼 Video 1 shows all slices of time frame 7. Identical images are indicated by the white squares. The lateral extent of the rectocele can be accurately evaluated by the extended spatial coverage of the newly proposed imaging technique. While banding artifacts are present in the bright fat tissues, none are visible within the patient's rectum.

\section{Discussion and Conclusion}

\section{$\nabla$}

The proposed $3 \mathrm{D}$ MR-defecography method offers the possibility to visualize the defecation process of patients with pelvic floor disorders with extended cov-
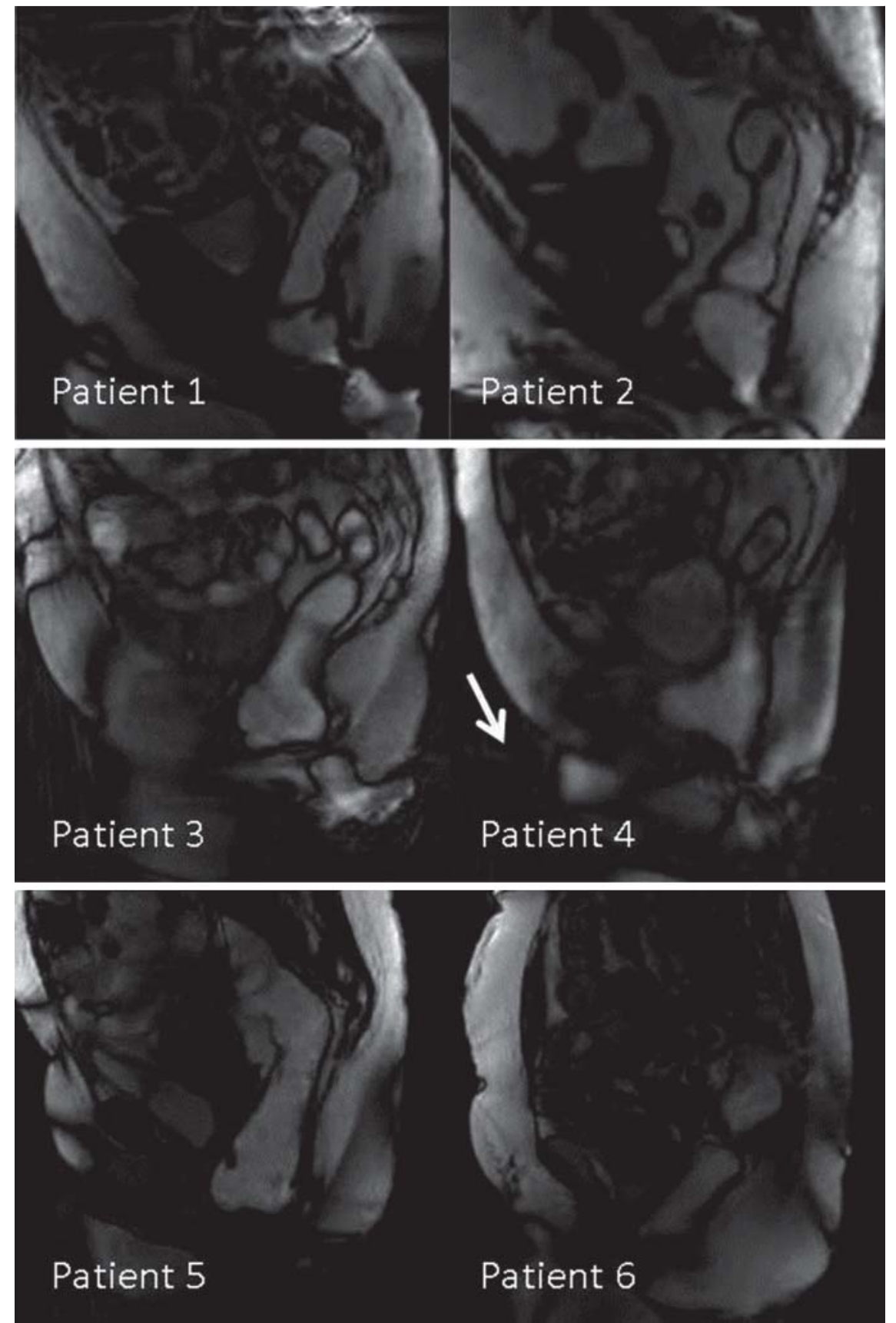

Fig. 2 A central slice of all patients. Patients 1 and 2 were examined without density weighting and view sharing (DW sampling 0), patients 3 and 4 were examined with DW sampling 1 and patients 5 and 6 were examined with DW sampling 2. Patients 5 and 6 feature the highest spatial resolution, the highest undersampling factors and nonetheless the highest image quality.

Abb. 2 Eine mittlere Schicht aller Patienten. Patient 1 und 2 wurden ohne „density-weighting“ und „view-sharing“ untersucht (DW-Sampling 0), die Patienten 3 und 4 mit DW-Sampling 1 und die Patienten 5 und 6 mit DW-Sampling 2. Die Patienten 5 und 6 besitzen die höchste räumliche Auflösung, die höchsten Unterabtastungsfaktoren und trotzdem die beste Bildqualität.

erage. Not only the anterior-posterior but also the lateral extent of a given pathology can be evaluated. Furthermore, the extended coverage provides more flexibility, because it is easier to angle a $3 \mathrm{D}$ volume properly than to angle a single 2 D slice. However, it has to be considered that the whole examination depends lar- gely on the individual patient and how strong the motion is during the defecation process.

We optimized the sampling scheme of our 3 D MR-defecography method to get optimized image quality with highly reduced data acquisition. We varied the acquisition parameters within an acceptable 

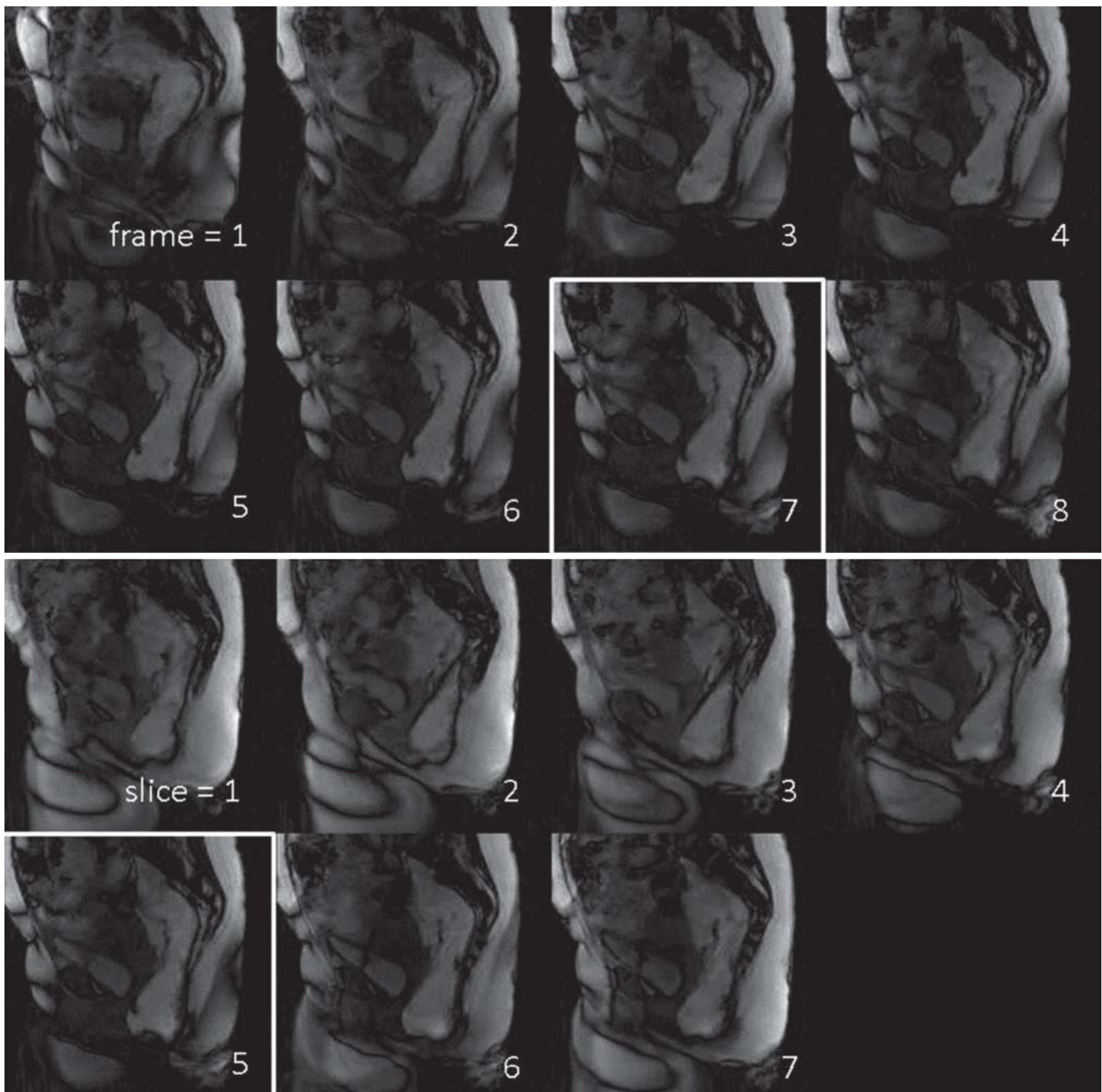

Fig. 3 Temporal progress of the defecation process of patient 5 (top). It is shown how a small anterior rectocele evolves during defecation. Due to the

Abb.3 (Oben) Zeitlicher Verlauf der Defäkation von Patient 5. Es ist zu sehen, dass sich während der Entleerung eine kleine anteriore Rektozele bildet. Durch die 3D-Messung ist es möglich die laterale Ausdehnung der Pathologie zu untersuchen. In der unteren Bilderserie sind alle Schichten von Bild 7 der oberen Bilderserie dargestellt. Identische Bilder sind durch weiße Rahmen gekennzeichnet. slices of the $3 \mathrm{D}$ block. At the bottom all slices of time frame 7 are shown. Identical images are indicated by white squares.

range regarding temporal and spatial resolution in 6 patients to determine an optimal trade-off. The first two patients showed that it is possible to visualize the defecation process with standard stackof-stars sampling. However, the sampling has three disadvantages.

First, only a small undersampling factor $\mathrm{R}=4$ was possible, because the standard stack-of-stars sampling has no variation in the $\mathrm{k}_{\mathrm{z}}$-direction and in this case a higher undersampling factor would result in severe undersampling artifacts. Second, the sampling doesn't consider the fact that the higher signal energy is located in the center of the $3 \mathrm{D} \mathrm{k}$-space and that missing data in this region leads to stronger artifacts in the reconstructed images than missing data in the $\mathrm{k}$-space periphery. Third, the number of reconstructed time frames can almost be doubled using the view sharing feature, which is reasonable for better dynamic visualization.

Therefore, we improved the sampling pattern with respect to these three points and adapted the two different DW sampling schemes. With the variation in the $\mathrm{k}_{\mathrm{z}}$-direction in combination with our $3 \mathrm{D}$ data reconstruction, higher undersampling factors were possible. For patient 3 we invested 
this time gain in a higher spatial resolution, which led to good image quality. For patient 4 we invested the time gain in an even higher image update rate than for patients 1 and 2. That, however, led to an increase in the artifact level that impaired diagnosis. Therefore, we further increased the spatial resolution and compensated the accompanying time loss with higher undersampling factors in the outer partitions
(DW sampling 2). This solution seemed to be the optimal trade-off of a good temporal and spatial resolution. An even higher spatial resolution would further prolong the scan time and higher undersampling factors would again increase the artifact level. Our proposed method shows that the fast, non-periodic, dynamic defecation process can be visualized with $3 \mathrm{D}$ MR imaging using a density-weighted and view- shared stack-of-stars sampling scheme in combination with a $3 \mathrm{D}$ FISTA compressed sensing reconstruction algorithm.

The next step comprises the comparison of our approach with the standard defecography methods in a larger patient collective.

V. Ratz, T. Wech, A. Schindele, A. Dierks, A. Sauer, J. Reibetanz, A. Borzi, T. Bley, H. Köstler, Würzburg, Germany 